\title{
Strain-relief pattern as guide for the formation of surface-supported bimolecular nanoribbons
}

\author{
K. Aït-Mansour, ${ }^{1,2, a)}$ M. E. Cañas-Ventura, ${ }^{1, b)}$ P. Ruffieux, ${ }^{1}$ R. Jaafar, ${ }^{1}$ M. Bieri, ${ }^{1}$ \\ R. Rieger, ${ }^{3}$ K. Müllen, ${ }^{3}$ R. Fasel, ${ }^{1}$ and O. Gröning ${ }^{1}$ \\ ${ }^{1}$ Empa, Swiss Federal Laboratories for Materials Testing and Research, nanotech@surfaces Laboratory, \\ Feuerwerkerstrasse 39, CH-3602 Thun, Switzerland \\ ${ }^{2}$ Ecole Polytechnique Fédérale de Lausanne (EPFL), Institut de Physique de la Matière Condensée, \\ Station 3, CH-1015 Lausanne, Switzerland \\ ${ }^{3}$ Max-Planck-Institut für Polymerforschung, Ackermannweg 10, D-55128 Mainz, Germany
}

(Received 3 August 2009; accepted 10 September 2009; published online 7 October 2009)

\begin{abstract}
We demonstrate the suitability of the $\mathrm{Ag} / \mathrm{Pt}(111)$ strain-relief pattern as efficient template for controlling the formation of well defined heteromolecular nanostructures. Two different species of molecular building blocks with complementary end-group functionalities are combined on this surface, which results in the formation of robust bimolecular nanoribbons driven by the interplay of the site specific adsorption on the strain-relief pattern with the highly directional intermolecular hydrogen-bonding intrinsic to the free bimolecular system. (c) 2009 American Institute of Physics. [doi:10.1063/1.3240891]
\end{abstract}

Specifically designed organic molecules can serve as basic units for the realization of functional components to be used in electronic, optoelectronic, or biochemical devices. ${ }^{1}$ To this ultimate end, it is crucial to control the assembly of individual molecules into predicted, highly ordered supramolecular structures. A straightforward and convenient way to do this in two dimensions consists of using atomically well defined surfaces of single crystals as supports, which nowadays constitutes an intensive research activity in nanoscience. ${ }^{2}$ Controlling the molecular arrangement and network formation is now possible by tuning intermolecular interactions, for instance via hydrogen-bonding following an adequate end-group functionalization of the molecules ${ }^{3,4}$ or metal coordination. ${ }^{5}$ The atomic structure of single-crystal surfaces with a typical periodicity of a few angstroms exhibits some templating function, e.g., in influencing the orientation of molecules. However, the challenge remains concerning the control of molecular adsorption sites with a periodicity of a few molecular diameters and a positional precision below $1 \mathrm{~nm}$, which might allow the realization of laterally controlled, specific hybrid molecular nanoassemblies. This challenge requires the use of template surfaces (i) showing laterally a strong modulation in the substratemolecule interaction and (ii) having a few $\mathrm{nm}$ periodicity, which is not accessible to conventional lithographic techniques. Examples of solid nanotemplates are the natural herringbone reconstruction of $\mathrm{Au}(111),{ }^{6}$ its vicinal surfaces ${ }^{7}$ and self-organized surface patterns occurring in heteroepitaxial systems, ${ }^{8,9}$ which have been previously used to influence the formation of molecular as well as atomic assemblies., ${ }^{4,9-17}$ The triangular strain-relief pattern formed by 2 monolayers (ML) of $\mathrm{Ag}$ on $\mathrm{Pt}(111)^{8}$ is particularly interesting because the bare terraces of this template surface show a high affinity for the site selective molecular adsorption and for the guidance of homomolecular nanostructures. ${ }^{14-17}$

In this letter, by investigating the case of a bimolecular system on $\mathrm{Ag} / \mathrm{Pt}(111)$, we demonstrate the possibility of con-

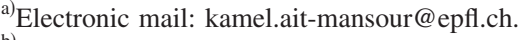

${ }^{b)}$ Present address: Kamerlingh Onnes Laboratory, Leiden University, P.O. Box 9504, 2300 RA Leiden, The Netherlands.
}

trolling the formation of surface structure-guided heteromolecular nanoassemblies from properly designed molecular building blocks. We associate two molecular species with complementary end-group functionalities, the 3,4,9,10perylenetetracarboxylic diimide (PTCDI) and the 1,4-bis(2,4-diamino-1,3,5,-triazine)-benzene (BDATB), and combine the bimolecular system related interaction with the laterally modulated substrate-molecule interaction on the strain-relief pattern. We therefore form well ordered and oriented, robust bimolecular nanoribbons whose (i) internal structure is governed by hydrogen bonds between the two molecular species and (ii) morphology is steered by the strain-relief pattern.

Experiments were carried out in ultra-high vacuum using a low-temperature scanning tunneling microscope $(\mathrm{STM})^{14-17}$ operated here at $77 \mathrm{~K}$. STM images were measured in the constant-current mode with the stated voltage referring to the electric potential of the sample with respect to the STM tip (mechanically cut from a Pt/Ir wire). The STM images have been processed with the wSXM software. ${ }^{18}$ The strain-relief pattern was prepared by evaporating $2 \mathrm{ML}$ $\mathrm{Ag}$ on a clean $\operatorname{Pt}(111)$ surface held at room temperature followed by annealing to $800 \mathrm{~K}^{14-17}$ PTCDI and BDATB molecules (Ref. 4) were simultaneously deposited on $\mathrm{Ag} / \mathrm{Pt}(111)$ kept at $370 \mathrm{~K}$ via sublimation from quartz crucibles resistively heated in a Kentax evaporator at about 660 and $470 \mathrm{~K}$, respectively. The STM images shown in this paper correspond to a molecular coverage of about 0.2 ML (PTCDI +BDATB) with a stoichiometry very close to 1:1.

Figure 1(a) shows an STM image of the $2 \mathrm{ML} \mathrm{Ag} /$ $\mathrm{Pt}(111)$ triangular pattern which originates from the $4.3 \%$ lattice mismatch between the bulk Ag and Pt crystal structures. This triangular pattern has a period of about $7 \mathrm{~nm}$ and contains in the rhombic unit cell one hexagonal fcc domain and two inequivalent triangular hcp domains, ${ }^{8}$ which we label as hcp1 and hcp2, with hcp1 being smaller than hcp2. The fcc and hep domains are separated by STM topographic depressions resulting from $\operatorname{Ag}\langle 1 \overline{1} 0\rangle$ oriented walls of Shockley partial dislocations ${ }^{8}$ probably buried in the underlying layers, which confers to the template surface a robust 


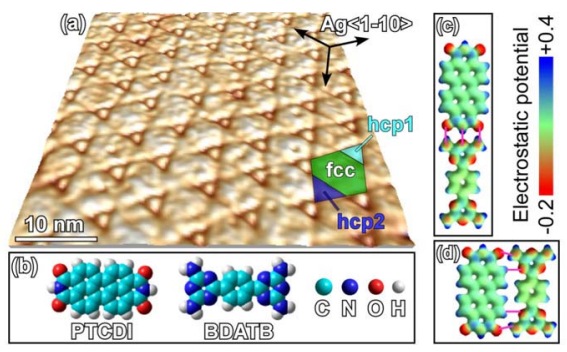

FIG. 1. (Color online) (a) Three-dimensional representation of an STM image of the $2 \mathrm{ML} \mathrm{Ag/Pt(111)} \mathrm{strain-relief} \mathrm{pattern} \mathrm{characterized} \mathrm{by} \mathrm{three}$ types of domains (fcc, hcp1 and hcp2) in the rhombic unit cell $(-0.5 \mathrm{~V}, 1$ nA). (b) Chemical structures of PTCDI and BDATB. [(c) and (d)] Calculated models of two hydrogen-bond configurations for PTCDI-BDATB pairs, (c) head-to-head and (d) side-to-side, where both molecules are shaded according to their electrostatic potential shown in color code in units of $e / a_{0}$. In [(c) and (d)] pink lines stand for H-bonds, and the center-tocenter intermolecular distances are 14.2 and $7.8 \AA$, respectively.

character. ${ }^{13}$ Figure 1(b) shows the chemical structures of the two molecular building blocks, PTCDI and BDATB, which were coevaporated on $\mathrm{Ag} / \mathrm{Pt}(111)$. The bimolecular system is characterized by complementary end-group functionalities with two - $\mathrm{CO}-\mathrm{NH}-\mathrm{CO}-$ (imide) groups having a hydrogenbonding acceptor-donor-acceptor sequence for the PTCDI and two -NH-N-NH- groups having a donor-acceptordonor sequence for the BDATB. ${ }^{19}$ Figures $1(\mathrm{c})$ and $1(\mathrm{~d})$ show force-field calculation ${ }^{20}$ models of two PTCDI-BDATB hydrogen-bonding configurations which can be predicted from the electrostatic potential of the two molecules calculated at the $\mathrm{AM} 1^{21}$ level, plotted in color code on a constant charge density contour of $0.05 e / a_{0}^{3}$ ( $a_{0}$ being the Bohr radius). The calculations show that the PTCDI-BDATB headto-head configuration displayed in Fig. 1(c), which leads to the formation of a triple hydrogen bond between the two molecular species, exhibits the strongest interaction energy of $E_{\mathrm{HH}}=922 \mathrm{meV}$ (attractive). The side-to-side configuration displayed in Fig. 1(d) is also attractive with an interaction energy of $E_{\mathrm{SS}}=334 \mathrm{meV}$. These calculation results show the highly directional character intrinsic to the free bimolecular system.

Codeposition of PTCDI and BDATB molecules onto the strain-relief pattern leads to the formation of well defined ribbon-like structures as shown in the STM image of Fig. 2(a). The strong correlation between the morphology of the ribbons and the triangular strain-relief pattern becomes immediately apparent when the hcp1 domains are highlighted, e.g., by cyan triangles, as in Fig. 2(b). At higher resolution [Fig. 3(a)] it can be seen that the ribbons are ordered on the template surface and linearly alternate PTCDI and BDATB molecules. Such molecular nanoribbons have been recently reported on the vicinal surface $\mathrm{Au}\left(\begin{array}{ll}11 & 12 \\ 12\end{array}\right)$, on which they are only guided by the regular, straight step edges. ${ }^{4}$ In the case of the $\mathrm{Au}(111)$ surface, however, the herringbone reconstruction of the bare terraces does not induce a sufficiently high modulation of the adsorption energy to allow any molecular ribbon formation. In this case the strong head-to-head and side-to-side hydrogen bonding leads to the formation of large binary islands. ${ }^{4}$ Also on the $\mathrm{Au}\left(\begin{array}{lll}11 & 12 & 12\end{array}\right)$ the discommensuration lines of the herringbone reconstruction running almost perpendicular to the step edges are not sufficient to break the PTCDI-BDATB ribbons into nanochains, ${ }^{4}$ as, e.g., in the case of $\mathrm{C}_{60}$ fullerenes on the same surface. ${ }^{11}$ Regarding now the present case of the $\mathrm{Ag} / \mathrm{Pt}$ template, the step
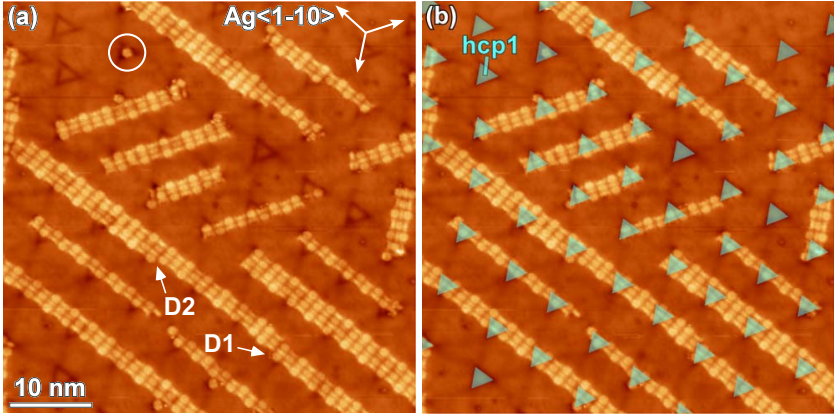

FIG. 2. (Color online) (a) STM image showing the formation/guidance of PTCDI-BDATB nanoribbons on a bare terrace of the strain-relief pattern $(-0.5 \mathrm{~V}, 0.05 \mathrm{nA})$. D1 and D2 stand for two different types of defects that can appear within the supramolecular structure of the nanoribbons. (b) Same STM image with cyan triangles highlighting the hcp1 domains.

edges are neither regular nor very straight; that is why we observe that they are randomly decorated by the molecules without any development of step-directed ribbons (not shown). Rather, as can be seen in Figs. 2 and 3(a), the PTCDI-BDATB ribbons form in the middle of the bare terraces, which shows that the strain-relief triangular structure induces a significantly stronger modulation of the adsorption energy than the $\mathrm{Au}(111)$ herringbone reconstruction.

The intermixing of the PTCDI and BDATB molecules in the nanoribbons is promoted by the strong interaction between the two molecular species via the triple hydrogenbonding as shown in Fig. 1(c). This heteromolecular interaction is much higher than the strongest interaction for homomolecular pairs of PTCDI and BDATB, by $37 \%$ and $85 \%$, respectively, ${ }^{4}$ which therefore makes phase separation between PTCDI and BDTAB less favorable. The linear character of the ribbons is also partially due to the triple hydrogen-bonding configuration along the ribbon axis. However it is evident that the triangular structure of the strainrelief pattern plays a crucial role in the formation/guidance of the heteromolecular ribbons. This is in line with our previous works demonstrating the high effectiveness of this template surface for controlling the formation of homomolecular $\left(\mathrm{C}_{60}\right.$, PTCDI) nanostructures. ${ }^{14-17}$ Significant work function variation from domain to domain on the strain-relief pattern is thought to be responsible for a more favorable molecular trapping in the hcp1 domains where the local work function is lowest. ${ }^{15}$ This is especially the case of the PTCDI molecule which, further known to be an electron acceptor, ${ }^{22}$ is expected to adsorb on low work function domains, as illustrated in the upper part of Fig. 2(a), where a single PTCDI is immobilized in an hep1 domain highlighted by a circle.

In addition to their high affinity to trap individual molecules, the hcp1 domains are actually those which guide the formation of the molecular ribbons. This is illustrated in Figs. 2 and 3 by the fact that (i) the ribbons in the vast majority of cases start and end at the hcp1 domains and (ii) they run in the three directions of the hexagonal lattice formed by the hcp 1 domains. In the STM images indication can be found that both the sequence of hcp1 domains and the $\mathrm{Ag}(111)$ atomic lattice are important for the morphology of the ribbons. To understand this, one needs to be aware that the triangular structure is not strictly regular, which can be seen in Fig. 1(a). This nonregularity is expressed by the fact that the edges of two nearest-neighbor hcp1 domains, which run along the same $\operatorname{Ag}\langle 1 \overline{1} 0\rangle$ direction, can be not strictly 

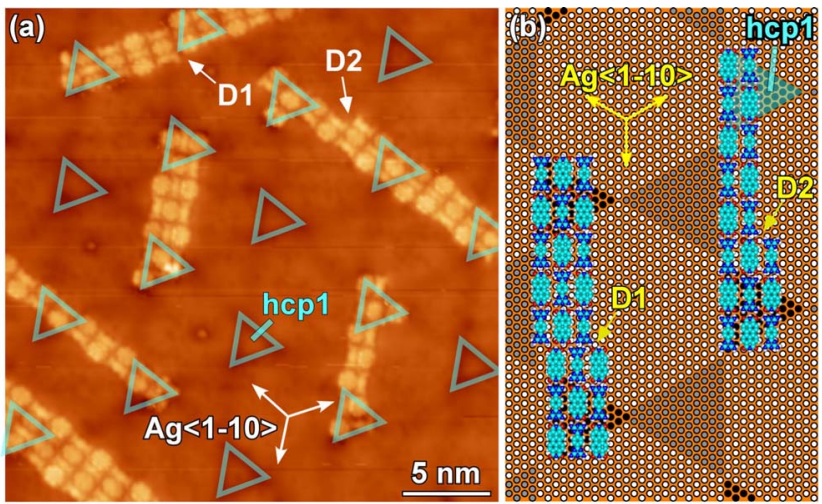

FIG. 3. (Color online) (a) Closeup STM image of the nanoribbons $(-0.5 \mathrm{~V}$, $0.05 \mathrm{nA}$ ). Triangles highlighting the hep1 domains show that the nanoribbons have one molecular chain which locks into an hcp1 edge. (b) Schematic representation of two-chain and three-chain ribbons on the strainrelief pattern. Are also represented the defects D1 and D2 shown in (a).

aligned but shifted by one or two atomic rows perpendicularly to this direction. In other words, the edges of the hcp1 regions always follow the $\operatorname{Ag}\langle 1 \overline{1} 0\rangle$ directions, which is not strictly the case for the direction defined by two neighboring hcp1 triangles. Nevertheless the ribbons follow this direction, and they do so not by a continuous distortion of their structure but by inclusion of discrete defects. Two principal types of defects labeled D1 and D2 are observed in Figs. 2(a) and 3(a) which are schematized in Fig. 3(b). D1 is a shift of the ribbon rows perpendicular to $\operatorname{Ag}\langle 1 \overline{1} 0\rangle$ by $5.1 \AA$ which corresponds the periodicity of the $\operatorname{Ag}(111)$ lattice along $\mathrm{Ag}\langle 11 \overline{2}\rangle$. Accordingly along the ribbon there is no shift of the molecule sequence within an error of $0.2 \AA$. D2 is even simpler than D1 and consists of starting, respectively ending, of molecular rows if they do, or do not, follow the sequence of the hcp1 domains. The nature of these defects which allow the ribbons to follow the hcp1 domains indicates that the molecules are strongly influenced by these domains and the $\operatorname{Ag}(111)$ lattice and are at least in orientational registry with the latter. In all cases the nanoribbons have one PTCDIBDATB chain which locks into the edge of an hep1 domain (Fig. 3).

The creation of D1 in the nanoribbons requires energy which can be estimated by force-field calculations. ${ }^{20}$ Considering a ribbon of three molecular chains (as frequently observed in the STM images), we have calculated the decrease in interaction energy at the D1 position as compared to the ideal head-to-head, three-chain structure, and we have found that it is of $884 \mathrm{meV}$ per D1 defect. As this defect is introduced in the ribbon so that the latter follows the hcp1 regions, one can estimate that this energy cost is at least compensated by the energy gain the ribbon experiences by covering the hcp1 domain. As the three-chain ribbons cover the hcp 1 regions almost totally, the number of molecules per hcp1 region, estimated from the average area of this region $\left(364 \AA^{2}\right)$ divided by the average area per molecule in the ribbons $\left(111 \AA^{2}\right)$, is roughly 3 . Therefore, by the formation of D1, the energy gain per heteromolecular pair in hcp1 is estimated to be at least $589 \mathrm{meV}$, which corresponds to $0.64 \times E_{\mathrm{HH}}$ [Fig. 1(c)] or $1.76 \times E_{\mathrm{SS}}$ [Fig. 1(d)], and therefore is significant and shows the strong adsorption anisotropy on the template surface. The stability of the heteromolecular ribbons has been tested by adsorbing foreign molecules on the sample, where we observe that the ribbons do not break up. This observation shows that the ribbons are robust on the bare terraces, a consequence of the highly directional PTCDI-BDATB hydrogen-bonding interactions and the strong molecular pinning on the strain-relief pattern.

In conclusion, we have used the $\mathrm{Ag} / \mathrm{Pt}(111)$ strain-relief pattern to steer specifically oriented robust biomolecular nanoribbons predefined from the highly directional hydrogenbonding interactions intrinsic to the bimolecular system. Our results demonstrate the suitability of this template for controlling with a nanoscale precision the formation of complex supramolecular assemblies with possible applications in molecular electronics depending on the molecule properties.

Financial support by the European Commission (RADSAS, NMP3-CT-2004-001561) is gratefully acknowledged.

${ }^{1}$ C. Joachim, J. K. Gimzewski, and A. Aviram, Nature (London) 408, 541 (2000); M. G. L. van den Heuvel and C. Dekker, Science 317, 333 (2007). ${ }^{2}$ J. V. Barth, Annu. Rev. Phys. Chem. 58, 375 (2007).

${ }^{3}$ J. A. Theobald, N. S. Oxtoby, M. A. Phillips, N. R. Champness, and P. H. Beton, Nature (London) 424, 1029 (2003)

${ }^{4}$ M. E. Cañas-Ventura, W. Xiao, D. Wasserfallen, K. Müllen, H. Brune, J. V. Barth, and R. Fasel, Angew. Chem., Int. Ed. 46, 1814 (2007).

${ }^{5}$ S. Stepanow, M. Lingenfelder, A. Dmitriev, H. Spillmann, E. Delvigne, N. Lin, X. Deng, C. Cai, J. V. Barth, and K. Kern, Nature Mater. 3, 229 (2004)

${ }^{6}$ J. V. Barth, H. Brune, G. Ertl, and R. J. Behm, Phys. Rev. B 42, 9307 (1990).

${ }^{7}$ S. Rousset, V. Repain, G. Baudot, Y. Garreau, and J. Lecoeur, J. Phys. Condens. Matter 15, S3363 (2003).

${ }^{8}$ H. Brune, H. Röder, C. Boragno, and K. Kern, Phys. Rev. B 49, 2997 (1994).

${ }^{9}$ R. Otero, Y. Naitoh, F. Rosei, P. Jiang, P. Thostrup, A. Gourdon, E. Lægsgaard, I. Stensgaard, C. Joachim, and F. Besenbacher, Angew. Chem., Int. Ed. 43, 2092 (2004).

${ }^{10}$ T. Yokoyama, S. Yokoyama, T. Kamikado, Y. Okuno, and S. Mashiko, Nature (London) 413, 619 (2001).

${ }^{11}$ W. Xiao, P. Ruffieux, K. Aït-Mansour, O. Gröning, K. Palotas, W. A. Hofer, P. Gröning, and R. Fasel, J. Phys. Chem. B 110, 21394 (2006).

${ }^{12}$ H. Brune, M. Giovannini, K. Bromann, and K. Kern, Nature (London) 394, 451 (1998).

${ }^{13}$ K. Aït-Mansour, A. Buchsbaum, P. Ruffieux, M. Schmid, P. Gröning, P. Varga, R. Fasel, and O. Gröning, Nano Lett. 8, 2035 (2008).

${ }^{14}$ K. Aït-Mansour, P. Ruffieux, W. Xiao, P. Gröning, R. Fasel, and O. Gröning, Phys. Rev. B 74, 195418 (2006).

${ }^{15}$ P. Ruffieux, K. Aït-Mansour, A. Bendounan, R. Fasel, L. Patthey, P. Gröning, and O. Gröning, Phys. Rev. Lett. 102, 086807 (2009).

${ }^{16}$ K. Aït-Mansour, P. Ruffieux, P. Gröning, R. Fasel, and O. Gröning, J. Phys. Chem. C 113, 5292 (2009).

${ }^{17}$ K. Aït-Mansour, M. Treier, P. Ruffieux, M. Bieri, R. Jaafar, P. Gröning, R. Fasel, and O. Gröning, J. Phys. Chem. C 113, 8407 (2009).

${ }^{18}$ I. Horcas, R. Fernández, J. M. Gómez-Rodríguez, J. Colchero, J. GómezHerrero, and A. M. Baro, Rev. Sci. Instrum. 78, 013705 (2007).

${ }^{19}$ L. J. Prins, D. N. Reinhoudt, and P. Timmerman, Angew. Chem., Int. Ed. 40, 2382 (2001).

${ }^{20}$ Calculations for heteromolecular pairs and ribbons have been performed using the AMBER force-field [W. D. Cornell, P. Cieplak, C. I. Bayly, I. R. Gould, K. M. Merz, D. M. Ferguson, D. C. Spellmeyer, T. Fox, J. W. Caldwell, and P. A. Kollman, J. Am. Chem. Soc. 117, 5179 (1995)] as integrated in HyperChem 7.5 (Hypercube Inc., $1115 \mathrm{NW}$ 4th Street, Gainesville, Florida 32601). In a first step all molecules involved in the calculations are relaxed individually at the AM1 level of theory (Ref. 21). Then for each configuration of the molecules the charge distribution is calculated using AM1, followed by the AMBER force-field calculation for the total energy. All calculations have been performed for gas-phase interactions with the effect of the surface being accounted for by forcing molecules to lie within the same plane.

${ }^{21}$ M. J. S. Dewar, E. G. Zoebisch, E. F. Healy, and J. J. P. Stewart, J. Am Chem. Soc. 107, 3902 (1985).

${ }^{22}$ M. Sassi, V. Oison, and J. M. Debierre, Surf. Sci. 602, 2856 (2008). 